\title{
Significado do trabalho e busca de emprego para jovens nem-nem
}

\author{
meaning of Work and Job Searching for Young neets \\ Significado del trabajo y de la búsqueda de empleo para jóvenes ninis
}

\author{
Daniele de Souza Paulino* \\ Pedro F. Bendassolli* \\ ${ }^{*}$ Universidade Federal do Rio Grande do Norte, Natal, Brasil.
}

Doi: http://dx.doi.org/10.12804/revistas.urosario.edu.co/apl/a.5112

\section{Resumo}

Esta pesquisa investigou a relação entre o significado do trabalho e comportamentos de busca de emprego para jovens nem-nem. Seu principal objetivo foi compreender se essas dimensões e o relacionamento entre elas ajudam a entender os motivos dos jovens estarem nessa condição. Participaram 224 jovens, que responderam a um questionário sociodemográfico, a Escala de Significados Atribuídos ao Trabalho, versão brasileira (ESAT-BR), a Escala de Intensidade de Busca de Emprego e itens sobre centralidade do trabalho. A análise dos dados provenientes das escalas foi precedida da realização de uma análise fatorial confirmatória (AFC), a qual propôs ajustes nos modelos originais. Os resultados das análises estatísticas apontam que jovens há mais tempo na condição nem-nem, tenderam a atribuir uma maior conotação negativa ao trabalho e a se envolverem menos em estratégias de busca de emprego. Jovens que concentravam os maiores níveis de escolaridade e de renda relataram maior envolvimento com as referidas estratégias. A associação encontrada entre varáveis sociodemográficas e os constructos investigados, evidenciam a complexidade do fenômeno e a importância de compreendê-lo a partir da interação entre fatores pessoais e contextuais.

Palavras-chave: jovens, centralidade do trabalho, significado do trabalho, desemprego.

\section{Abstract}

This research studied the relationship between the meaning of work and job search behaviors for young people who are not in education, employment, or training (NEETs). Its main objective was to understand whether these dimensions and the relationship between them explain the reasons why NEETs are in this condition. In total, 224 young people participated in the study and answered a personal and social questionnaire, an ESAT-BR, job-search behaviors intensity scale and items

* Daniele de Souza Paulino, Pedro F. Bendassolli, Departamento de Psicologia, Universidade Federal do Rio Grande do Norte, Natal, Brasil.

Correspondência a respeito deste artigo deve ser endereçada para Daniele de Souza Paulino, Av. Senador Salgado Filho, s/n, Campus Universitário, CEP 59078-970, Natal, Brasil. Correio eletrônico: paulino223@gmail.com

Cómo citar este artículo: Paulino, D. S., D. \& Bendassolli, P. F. (2018). Significado do trabalho e busca de emprego para jovens nem-nem. Avances en Psicología Latinoamericana, 36(2), 373-388. DoI: http://dx.doi.org/10.12804/revistas.urosario. edu.co/apl/a.5112 
about the centrality of work. Analysis of data from the scales was preceded by a confirmatory factorial analysis (CFA), which proposed adjustments in the original models. The results point out that young people have been in the NEET condition for longer, tended to attribute a greater negative connotation to work, as well as have less involvement in job search strategies. At the same time, those who concentrated the highest levels of schooling and income, reported more involvement with these strategies. The association between personal and social variables and the researched constructs demonstrate the complexity of the phenomenon and the importance of understanding it from the perspective of the interaction between personal and contextual factors. Keywords: young adults, work centrality, meaning of work, unemployment.

\section{Resumen}

Este estudio investigó la relación entre el significado del trabajo y de los comportamientos de búsqueda de empleo para jóvenes ninis. Su principal objetivo fue entender si estas dimensiones y la relación entre ellas ayudan a comprender los motivos por los cuales los jóvenes continúan en esta situación. Participaron 224 jóvenes que respondieron a un cuestionario personal y social, a la Escala de Intensidad de la Búsqueda de Empleo (ESAT-BR), y a preguntas sobre la centralidad del trabajo. El análisis de los datos provenientes de las escalas fue precedido de la realización de un análisis factorial confirmatorio (AFC), el cual propuso ajustes en los modelos originales. Los resultados de los análisis estadísticos apuntan a que los jóvenes que llevan más tiempo en la condición nini tienden a atribuir una mayor connotación negativa al trabajo y a involucrarse menos en estrategias de búsqueda de empleo. Al mismo tiempo, aquellos que concentraban los mayores niveles de escolaridad y de renta relataron mayor implicación con dichas estrategias. La asociación encontrada entre variables personales y sociales, y los constructos investigados evidencian la complejidad del fenómeno y la importancia de comprenderlo a partir de la interacción entre factores personales y contextuales.
Palabras clave: jóvenes, centralidad del trabajo, significado del trabajo, desempleo.

A juventude foi considerada durante muito tempo uma fase de transição psicossocial para a idade adulta, representada pela preparação escolar e a consequente inserção no mercado de trabalho. Essa forma de conceber esse momento do ciclo vital era correlata de uma realidade socioeconômica e cultural marcada pela estabilidade - uma das características do mercado de trabalho até meados da década de 1970. No entanto, a crise do capitalismo que se sucedeu por volta desse período, assentada em larga reestruturação produtiva (Harvey, 2009), provocou, pouco a pouco, uma série de transformações no contexto laboral, as quais acabaram repercutindo sobre os jovens (Blanch, 2014). Nesse novo contexto, caracterizado pela flexibilidade, terceirização, precariedade e desemprego, a relação do jovem com o trabalho se tornou múltipla e complexa, marcada pela heterogeneidade das trajetórias laborais, devido à dificuldade de acesso ao emprego (Pochmann, 2007).

Nas últimas décadas, a inserção do jovem no mundo do trabalho transformou-se em um percurso árduo, marcado pela alternância de períodos de desemprego, inatividade e reinserções, geralmente em trabalhos precários e desprotegidos (Pais, 2016). Paralelamente, o investimento nas qualificações profissionais e o aumento do nível de escolaridade parecem não mais garantir a inserção e a estabilidade ocupacional. Nesse contexto, o primeiro contato do jovem com o trabalho passou a ser marcado de um lado por oportunidades temporárias e informais, e de outro, pelo desemprego e pela realidade da não inserção profissional (Coelho, Estramiana \& Luque, 2014). Com relação a essa última, um fenômeno tem ganhado cada vez mais repercussão midiática: o do jovem que, por diversas razões, abandona o desejo de trabalhar e também o de estudar. Na literatura, tal fenômeno é denominado de "nem-nem" e agrega sob a mesma terminologia uma variedade de situações, desde o desemprego temporário e a espera por melhores 
oportunidades profissionais, até o desemprego por longo prazo, e a decisão de não trabalhar (Blanch, 2014; Escobedo, 2013; Serracant, 2014).

Diante do exposto, quais poderiam ser os motivos ou as explicações para essa decisão de não trabalhar nem estudar, observada em contingente crescente de jovens? Segundo o Instituto Brasileiro de Geografia e Estatística, em 2015, estimou-se que no Brasil, 23,6\% dos jovens com idade entre 18 e 24 anos não estudava e nem trabalhava. No estado atual da literatura sobre o tema, em que pese sua incipiência, não há consenso sobre os determinantes do jovem nem-nem. Por exemplo, há autores que atribuem ao jovem a responsabilidade por sua condição, seja por desengajamento com as principais instituições sociais, por problemas emocionais e comportamentais ou devido ao envolvimento em atividades criminosas (e.g., Benjet et al., 2012; Seddon, Hazenberg \& Simon, 2013). De acordo com essa abordagem, faltaria ao jovem a motivação e a autoestima necessárias para procurar um emprego e oportunidades educacionais (Simmons \& Thompson, 2014).

Em contrapartida, autores como Cardoso (2013) e Vargas-Valle e Cruz-Piñeiro (2012), defendem uma perspectiva que compreende o fenômeno nem-nem a partir da interação entre fatores pessoais e contextuais. Assim, embora os fatores subjetivos sejam importantes para a compreensão na determinação do fenômeno em questão, eles devem ser interpretados com a ajuda dos contextos sociais em que estão inseridos os jovens. Essa dimensão contextual inclui aspectos como o acesso à oportunidades profissionais e de educação, e as características do contexto macroeconômico, os quais são marcados por diversos tipos de desigualdade.

Neste artigo, gostaríamos de destacar um fator em específico, relacionado ao significado do trabalho. Nessa direção, Blanch (2014) questiona sobre se tal significado não poderia ser um fator psicossocial capaz de contribuir na explicação da emergência do jovem nem-nem. O autor supracitado considera que os valores e representações sobre o trabalho estejam em mutação. De categoria central nos processos de socialização dos jovens e, principalmente, elemento decisivo capaz de marcar o ritual de passagem para a vida adulta, o trabalho tem, a despeito de sua centralidade econômica, disputado espaço com outras fontes de sentido na contemporaneidade, especialmente no universo juvenil. De acordo com Blanch (2014), a existência de jovens nem-nem poderia estar evidenciando uma transformação silenciosa nos significados atribuídos ao trabalho, principalmente na concepção do trabalho enquanto atividade central na vida das pessoas. Nesse ponto, e considerando a importância desse tema neste artigo, nos dedicamos a explorar brevemente a literatura sobre significado do trabalho.

Enquanto campo de investigação, as primeiras pesquisas sobre os significados do trabalho foram realizadas ainda na década de 1970 . Impulsionadas pelas mudanças que estavam acontecendo no mundo do trabalho -marcadamente, a expansão do desemprego, da informalidade e dos contratos atípicos de trabalho, cada vez mais flexíveis e precários- essas investigações buscavam captar as repercussões desses processos sobre os significados atribuídos ao trabalho, partindo da premissa de que tais significados estão umbilicalmente ligados ao contexto e às formas de institucionalização do trabalho. Desde então, diversas áreas do conhecimento, e entre elas a psicologia, têm desenvolvido aproximações teórico-conceituais sobre a dimensão do significado (Schweitzer, Gonçalves, Tolfo \& Silva, 2016).

Dentre essas aproximações, a perspectiva cognitivista desenvolvida a partir da pesquisa realizada pelo Meaning of Work International Research Team ou MOW, em 1987, reconhece o significado do trabalho como um constructo multifacetado, dinâmico e multidimensional. Dessa forma, o significado atribuído ao trabalho se dá a partir da confluência de aspectos históricos, dinâmicos e subjetivos. Logo, a percepção individual sobre essa esfera de vida também é fruto dos significados 
compartilhados em determinado contexto social e histórico, sendo, portanto, interpretações em constante construção (MOW, 1987).

Na composição do modelo do MOW (1987), uma dimensão tem recebido, ao longo dos anos, maior destaque investigativo: a dimensão da centralidade do trabalho, a qual consiste de uma classificação sobre a importância, afetiva e cognitiva, atribuída ao trabalho - seja em termos absolutos ou relativos, comparando-se o trabalho com outras atividades da vida (Harpaz, Honig \& Coetzier, 2002). Para Kanungo (1982), cujo instrumento foi utilizado nesta pesquisa, a centralidade do trabalho é uma crença normativa sobre a importância dessa esfera para uma pessoa, construída a partir de elementos culturais e de experiências prévias.

Em uma perspectiva semelhante, Gonçalves (2006) considera que os significados atribuídos ao trabalho se desenvolvem graças ao aparato psicossocial de cada pessoa, constituído por elementos históricos e culturais compartilhados no contexto social de pertença. Como resultado, as interpretações individuais acerca do trabalho são fruto tanto das experiências pessoais do sujeito com essa esfera de vida, quanto da relação que estabelece com diferentes atores sociais, por meio dos quais ela tem acesso à informações, opiniões, crenças e valores sobre o trabalho. Nessa perspectiva, o ser humano não é um mero reagente aos estímulos do meio, mas um construtor ativo de significados (Fernandes, Gonçalves \& Oliveira, 2012).

Pesquisas sobre significado do trabalho para jovens revelam que o trabalho tende a ser uma atividade valorizada, na medida em que é uma fonte de interação social, autoestima e prestígio, além de ser um canal de expressão de habilidades e competências, pelos quais os jovens se sentem reconhecidos e valorizados (Fernandes et al., 2012). No entanto, tal como elucidado por Mota e Tonelli (2013) em uma pesquisa com jovens de diferentes segmentos juvenis, um significado em particular parece se destacar: o de ser o trabalho uma atividade meramente instrumental. Para os jovens pesquisados, essa atividade é compreendida como um meio de se ganhar dinheiro e adquirir bens, tanto para atender suas necessidades de consumo, quanto para ajudar a família.

Essa relação instrumental com o trabalho também é citada entre as justificativas que os jovens utilizam para classificar o trabalho, enquanto uma dimensão de importância em suas vidas. Nessa perspectiva, Mota e Tonelli (2013) destacam que jovens de baixa condição socioeconômica avaliam o trabalho como central pelo fato de este lhes proporcionar renda e lhes dar acesso ao consumo. Assim, para esses jovens o trabalho é associado à possibilidade de mudar sua realidade social e financeira (Dutra-Thomé \& Koller, 2014). Devido à necessidade familiar, muitos deles se veem impelidos a abandonar os estudos precocemente para se inserirem em atividades de trabalho precárias, informais e mal remuneradas. Mesmo assim, veem no emprego a oportunidade de se sentirem mais produtivos, e de terem acesso a bens que seus pais muitas vezes não podem lhes proporcionar (Otero, 2011).

Embora com menor frequência, a percepção negativa sobre o trabalho também é citada por jovens em algumas pesquisas. Por exemplo, o trabalho é associado à exploração, sacrifício, dificuldades e a condutas abusivas (Dutra-Thomé \& Koller, 2014). Essa percepção negativa também é construída a partir das experiências de trabalho anteriores vivenciadas por esses jovens, nas quais eles são submetidos a situações de exploração e pouco reconhecimento profissional (Rocha-deOliveira, Piccinini \& Silveira, 2010).

Para além desses fatores psicossociais, como é o caso do significado do trabalho e seu papel na explicação do fenômeno, outras pesquisas têm buscado desenvolver estratégias pragmáticas para o enfrentamento da situação, com particular interseção com as políticas públicas e as ações do Estado (Costa \& Ulyssea, 2014). Vamos destacar na sequência, pesquisas voltadas à investigação das estratégias de inserção utilizadas pelos jovens, em 
uma tentativa de acercar o fenômeno a partir de algumas possíveis formas de enfrentamento dessa situação. Considera-se nessa tentativa, a superação das potenciais barreiras psicossociais que levam ao abandono do desejo de trabalhar ou de estudar.

Como campo de estudo, as pesquisas sobre busca de emprego têm se concentrado em investigar elementos preditores e os resultados dessas estratégias, além de propor intervenções que auxiliem as pessoas no comportamento de busca e na consecução de uma atividade de trabalho. Os comportamentos de busca de emprego podem se diferenciar a partir da utilização de determinados recursos. Em um primeiro eixo, eles se realizam a partir da própria iniciativa da pessoa em procurar oportunidades de trabalho, seja por meio da entrega de currículos, leitura de classificados, entre outros (Magalhães \& Teixeira, 2013). Por outro lado, a busca de emprego pode sofrer a interferência da rede de contatos das pessoas. Por meio dessa rede, as pessoas conseguem informações, dicas e conselhos sobre as oportunidades de trabalho existentes, e sobre como conseguir um emprego (Wanberg, Kanfer \& Banas, 2000).

Para Guimarães, Andrada, Picanço e Vieira (2012), o tamanho da rede social e a qualidade das informações por ela disponibilizadas se diferenciam com base na classe social. Em seu estudo, jovens egressos do ensino superior provenientes de famílias com rendimentos de até 3 salários mínimos, tinham seus familiares como principal canal de acesso ao emprego. Nesse contexto, as oportunidades de emprego são caracterizadas pela precariedade e baixos rendimentos. Por outro lado, jovens de classes socioeconômicas mais altas possuíam redes de contato mais amplas, formadas por sua família e pelo círculo de amigos. Nesse estudo, a renda relacionou-se à possibilidade de se conseguir uma ocupação de melhor qualidade.

Associada à renda, o nível de escolaridade também influencia os comportamentos de busca de emprego. Kanfer, Kantrowitz e Wanberg (2001) identificaram que jovens com menor nível de escolaridade tenderam a relatar pouco envolvimento com os comportamentos de busca de emprego. Para Šverko, Galić, Seršić e Galešić (2008), tal fator, associado aos poucos recursos financeiros e a percepção do sujeito de que sua escolaridade não é suficiente para atender as exigências demandadas pelo mercado de trabalho, ajudam a explicar essa menor procura por emprego. Ademais, Dorsett e Lucchino (2012) salientam que o tempo na condição nem-nem é um fator de risco, uma vez que longos períodos estão associados a menor intensidade de procura por emprego, o que dificulta a saída do jovem da mesma, caracterizando, dessa forma, um ciclo de difícil superação.

Diante do exposto, o presente artigo tem como objetivo contribuir para a compreensão dos fatores relacionados à juventude nem-nem. Para analisar esse fenômeno, e alcançar esse objetivo, o artigo, derivado de pesquisa empírica, elege duas grandes frentes teóricas e operacionais: a relação entre o significado e a centralidade do trabalho para jovens classificados como nem-nem, e a mobilização, também por estes jovens, de estratégias objetivas de busca de emprego. Também se verificou se esse fenômeno ocorre na vida desses jovens e quando ele ocorre. Desse modo, são caracterizados a seguir, os aspectos metodológicos dessa investigação.

\section{Método}

\section{Participantes}

Participaram da pesquisa pessoas com idade entre 18 e 24 anos, que não estavam desenvolvendo atividades registradas em carteira de trabalho e/ou informais, nem associadas à iniciativas educacionais ou de qualificação. Seguindo esses critérios, participaram do estudo 224 jovens. Do número total de respondentes, 75,9\% eram mulheres. A média de idade dos participantes foi de 21,24 anos $(D P=2,09)$. Quanto aos rendimentos, eles se concentraram em 1 salário $(28,6 \%)$, seguido por 2 a 3 salários mínimos (17\%). O nível 
de escolaridade mais relatado foi o ensino médio completo $(27,7 \%)$, seguido do ensino superior completo $(23,7 \%)$. Sobre os aspectos relacionados à experiência profissional dos jovens, 60,3\% já trabalharam em algum momento de suas vidas, embora sem registro em carteira de trabalho (47,8\%). Em média, o tempo que passaram nas ocupações foi de 11,23 meses $(D P=3,53)$. Por último, quanto ao tempo na condição nem-nem, $45,5 \%$ relataram estar nela entre 10 e 24 meses, enquanto que $31,7 \%$ afirmaram estar nessa condição entre 34 e 72 meses.

\section{Instrumentos}

A coleta de dados foi realizada a partir de um conjunto de questionários. O primeiro deles contemplou aspectos de caraterização pessoal e sociodemográfica. Os itens propostos foram elaborados a partir do levantamento bibliográfico sobre a juventude nem-nem, fazendo referência a aspectos como renda, escolaridade, experiência de trabalho, tempo na condição e contexto familiar.

As questões sobre o significado do trabalho foram investigadas a partir da Escala de Significados Atribuídos ao Trabalho (Abreviada), versão brasileira (ESAT-BR) (Fernandes et al., 2012), coerente com o referencial teórico apresentado na introdução, inscrito na tradição da equipe MOW (1987). O instrumento, originalmente desenvolvido em Portugal por Gonçalves (2006), foi adaptado e validado entre estudantes do ensino médio brasileiro por Fernandes et al. (2012). Durante o processo de adaptação, a escala passou a contar com 25 itens distribuídos entre quatro fatores: (a) Dimensão da Realização Pessoal do Trabalho, com 9 itens $(\alpha=0,81)$; (b) Dimensão Positiva do Trabalho, com 7 itens $(\alpha=0,77)$; (c) Dimensão Econômica do Trabalho ( $\alpha=0,64)$, com 3 itens; e (d) Dimensão Negativa do Trabalho, com 6 itens $(\alpha=0,77)$. As respostas aos itens são dadas em uma escala Likert de 6 pontos (Discordo totalmente a Concordo totalmente).
A centralidade do trabalho foi investigada por meio da Escala de Centralidade do Trabalho de Kanungo (1982), adaptada para o contexto brasileiro no estudo de Bendassolli, Alves e Torres (2014). Essa medida possui 6 itens e avalia a crença geral sobre o valor do trabalho na vida de uma pessoa e seu envolvimento com ele, por meio de uma escala Likert de 5 pontos, que varia de Discordo totalmente a Concordo totalmente $(\alpha=0,80)$. A esse instrumento, foram acrescentadas 2 questões propostas pelo MOW (1987): uma correspondente à centralidade absoluta do trabalho, na qual é solicitado que o respondente assinale, em uma escala de 1 a 5 (Sem importância a Extremamente importante), o grau de importância do trabalho em sua vida; e outra referente à centralidade relativa, que solicita que o participante distribua 100 pontos (cumulativos) entre as seguintes esferas de vida: ócio, comunidade, trabalho, religião e família. $\mathrm{O}$ acréscimo dessas duas questões permitiu que fosse identificado o lugar que o trabalho ocupa na vida dos jovens, quando considerados outros domínios existenciais.

Por fim, os comportamentos de busca de emprego foram mensurados por meio da Escala de Intensidade de Contatos de Rede Social e da Escala de Intensidade Geral de Busca de Emprego. Ambas foram desenvolvidas por Wanberg et al. (2000) e adaptadas ao contexto brasileiro por Magalhães e Teixeira (2013) em uma pesquisa com estudantes universitários. A primeira é composta por 6 itens que descrevem o comportamento de busca de emprego com o uso de redes de apoio social, cuja função é fornecer informações, indicações, dicas, entre outros $(\alpha=0,86)$. Por sua vez, a segunda é formada por 7 frases que descrevem a busca por postos de trabalho, sem o uso dessas redes (e.g., entrega de currículo, busca na internet, contato com órgãos de intermediação de mão-de-obra) $(\alpha=0,88)$. Em ambas, os respondentes assinalam em uma escala Likert de 5 pontos, a frequência da realização dessas atividades nos últimos 30 dias (Nunca/nenhuma vez a Muito/Mais de 10 vezes). 


\section{Procedimentos de coleta de dados e cuidados éticos}

Foram utilizadas duas estratégias de coleta, uma presencial e outra on-line. A primeira foi conduzida em diferentes bairros de duas cidades do Estado do Rio Grande do Norte, Brasil. A escolha por esses bairros se deu a partir de um critério de conveniência, especificamente o de acessibilidade às unidades básicas de saúde (UBSs). A opção por acessar os jovens por meio dessas unidades de saúde foi fundamentada na clara inserção que seus profissionais possuem nas respectivas comunidades, especificamente o agente comunitário de saúde. Dentre as atividades desenvolvidas por esse profissional, figuram as visitas domiciliares diárias e o acompanhamento das necessidades de saúde das pessoas de cada casa. Diante da natureza desse trabalho, o agente foi pensado como um parceiro que viabilizaria o acesso a respondentes em potencial para a pesquisa.

Na primeira etapa do processo, após ciência dos critérios de inclusão dos participantes, os agentes realizaram um breve levantamento dos prontuários das famílias residentes em suas áreas de atuação. Ao identificar as casas com jovens na situação nem-nem, eles guiaram a pesquisadora até as mesmas. O primeiro contato com o jovem era realizado pelo próprio agente de saúde, que apresentava a pesquisadora. Em seguida, esse profissional seguia para as suas atividades específicas com os outros membros da família, enquanto que a aplicadora do questionário explicava ao jovem os objetivos da pesquisa. Ao decidir participar, os aspectos do Termo de Consentimento Livre e Esclarecido (TCLE) foram explicitados e o jovem convidado a assinar o TCLE em duas vias. A aplicação do questionário foi realizada pela própria pesquisadora, que lia as afirmativas para o jovem e assinalava suas respostas. Isso ocorreu devido ao tempo limitado de cada visita realizada pelo agente, que durava em média 20 minutos. Com o intuito de minimizar a influência do pesquisador nas respostas as questões, buscou-se padronizar a explicação das orientações de cada questionário e a leitura dos seus itens.

A estratégia on-line transcorreu de forma paralela a anterior, tendo em vista a necessidade de se obter uma maior quantidade de questionários. Nesse caso, a Universidade Federal do Rio Grande do Norte (UFRN) emitiu um e-mail para alunos egressos dos últimos 5 anos, com o convite para que aqueles que atendessem aos critérios pudessem participar. A escolha por essa universidade se deu a partir de um critério de conveniência (facilidade de acesso da pesquisadora). O questionário estava hospedado no Google Forms e o link foi encaminhado junto ao e-mail convite. Na página inicial, o respondente teve acesso a um TCLE, que explicou os objetivos da pesquisa e garantiu o anonimato e o direito a retirar a participação a qualquer momento.

A coleta de dados foi iniciada, em ambos os casos, após a anuência das Secretárias de Saúde dos municípios e dos diretores das UBSs, no primeiro caso; e da UFRN no segundo, como também da aprovação do Comitê de Ética em Pesquisa, por meio do protocolo de número 47387515.8.0000.5537.

\section{Procedimentos de análise de dados}

Os dados provenientes das duas estratégias de coleta foram organizados em uma planilha Excel, o que permitiu a identificação de respostas ausentes e a verificação do atendimento aos critérios de participação na pesquisa, em particular no caso das respostas on-line. Após esse procedimento, foram identificados 224 questionários válidos para a análise. Em seguida, os dados foram exportados para o software IBM Statistical Package for the Social Sciences 20 (SPSS), e submetidos a análises de frequência, análises bivariadas com o intuito de atestar correlações entre os fatores de significado do trabalho, e deles com algumas variáveis sociodemográficas de interesse do estudo $\left(r_{s} \mathrm{de}\right.$ Spearman). Também utilizamos os testes $U$, de 
Mann-Whitney, e o de Kruskal-Wallis, para verificar diferenças entre grupos de respondentes.

Esses testes estatísticos foram utilizados devido à constatação de violação aos critérios de normalidade na amostra. A curtose e a assimetria oscilaram de $|22,19|$ e $|2,02|$ e de $|-4,58|$ a $|0,83|$, respectivamente, para a ESAT-BR; de $|-1,61|$ a $|-1,25|$ e de $|-0,59|$ a $|0,35|$, respectivamente, para a Escala de Centralidade do Trabalho; e de |-1,42| a |2,29| e de $|1,88|$ a $|0,42|$, respectivamente, para a Escala de Intensidade de Busca de Emprego. Esse desvio da normalidade ainda foi confirmado por meio da análise dos histogramas.

No entanto, antes da realização das análises supracitadas, a versão original da ESAT-BR e da Escala de Intensidade de Busca de Emprego foram submetidas à análise fatorial confirmatória $-\mathrm{AFC}-\mathrm{com}$ o auxílio do software AMOS $\AA 21.0$, e utilização do método Maximum Likelihood. Essa decisão se fundamenta no fato de que ambas já foram validadas em estudos anteriores para públicos jovens semelhantes ao desta pesquisa (Fernandes et al., 2012; Magalhães \& Teixeira, 2013) - portanto, o objetivo da AFC foi confirmar os modelos originalmente propostos nessas investigações, antes de utilizá-los em análises subsequentes. Como se estipula na literatura, tais análises são robustas ao ponto de não serem severamente afetadas por problemas de normalidade amostral (e.g., Kline, 2016).

Com o intuito de aferir a qualidade de ajuste geral dos modelos, foram utilizados alguns índices e valores propostos por Balbinotti, Balbinotti, Marques e Gaya (2009) e Hair, Anderson, Tatham e Black (2009). Entre eles, destacam-se as medidas de ajuste parcimonioso $\left(\chi^{2} / \mathrm{gl}<3\right.$, GFI $\geq 0,85$, AGFI $\geq 0,80$, AIC e CAIC: valores menores são preferíveis), medidas de ajuste incremental (TLI $\geq 0,90$ e CFI $\geq 0,90$ ) e medidas de ajuste absoluto (RMSEA $<0,05$ e SRMR $<0,008)$.

Além desses, com o intuito de verificar o ajuste dos modelos, no que se refere à mensuração dos constructos, foram observadas: a validade convergente, por meio da variância média extraída
$(\mathrm{VME} \geq 0,50)$ e da confiabilidade composta ( $\mathrm{CC} \geq$ $0,70)$; a validade discriminante, aferida por meio do valor da correlação entre as dimensões $(r \leq$ 0,80 ) e da raiz quadrada da variância extraída (raiz quadrada da $\mathrm{VME} \geq r$ ) (Brown, 2014; Hair et al., 2009; Kline, 2016). Também foram consideradas a validade fatorial dos modelos (cargas padronizadas $>0,40)$ e o Alfa de Cronbach $(\alpha \geq 0,70)$ para as escalas e seus fatores, de modo a constatar sua consistência interna - fidedignidade (Field, 2009).

\section{Resultados}

A tabela 1 apresenta os índices dos critérios de qualidade de ajuste dos dois modelos da ESAT-BR. Após a submissão do modelo original dessa escala à $\mathrm{AFC}$, foi possível verificar que dois itens apresentaram cargas fatoriais inferiores a 0,40 (Q7: “A possibilidade de realizar atividades de lazer como: esporte, convívio com os amigos, participação em clubes"; e Q13: "Cada vez maior exigência, dedicação e empenho"). Com relação aos critérios de validade discriminante, foi identificada a existência de uma correlação fatorial importante, superior a 0,80 , entre a Dimensão Positiva do Trabalho e a Dimensão Econômica do Trabalho $(r=0,86)$, evidenciando uma possível baixa capacidade discriminativa dos mesmos e a sobreposição entre esses fatores. Por último, a análise de confiabilidade dos fatores da escala atestou que essa última dimensão apresentou valor de alfa de 0,69 , inferior ao tradicionalmente aceito como mínimo.

A partir dessas constatações, foram realizadas modificações no modelo. Especificamente, os dois itens que apresentaram carga fatorial inferior a 0,4 foram excluídos, e os itens da Dimensão Econômica do Trabalho foram incorporados a Dimensão Positiva, modificação essa que possibilitou a redução da quantidade de fatores de 4 para $3 . \mathrm{Na}$ etapa que se seguiu, os 23 itens foram mantidos, pois todos apresentaram cargas fatoriais superiores ao valor estipulado. 
Tabela 1

Critérios de qualidade de ajuste da ESAT-BR

\begin{tabular}{crr}
\hline Critérios & \multicolumn{1}{c}{ M1 } & \multicolumn{1}{l}{ M2 } \\
\hline$\chi^{2}$ & 540,388 & 383,609 \\
Gl & 269 & 222 \\
$\chi^{2} / \mathrm{gl}$ & 2,00 & 1,728 \\
GFI & 0,837 & 0,871 \\
AGFI & 0,837 & 0,840 \\
AIC & 652,388 & 491,609 \\
CAIC & 899,440 & 729,838 \\
TLI & 0,854 & 0,908 \\
CFI & 0,869 & 0,919 \\
RMSEA & 0,067 & 0,057 \\
SRMR & 0,063 & 0,062 \\
\hline
\end{tabular}

Nota. M1: Modelo original; M2: Modelo final.

Em seguida, verificamos os índices dos critérios de qualidade de ajuste. De forma geral, em um primeiro momento, esses ficaram abaixo dos valores aceitáveis e foram inferiores aos encontrados no modelo original (M1): $\chi^{2} / \mathrm{gl}=2,163, \mathrm{p}<$ 0,$001 ;$ RMSEA $=0,072 ;$ SRMR $=0,065 ;$ TLI $=0,853$; $\mathrm{CFI}=0,868 ; \mathrm{GFI}=0,841 ;$ e AGFI $=0,807$. Diante disso, foram realizadas as alterações sugeridas pelos índices de ajustamento do software, com o objetivo de aperfeiçoar o modelo. Especificamente, foram ligadas 5 duplas de erros. Essa decisão foi fundamentada em justificativas teóricas e na proximidade semântica entre os itens.

Com a ligação entre os erros, houve um aperfeiçoamento geral do modelo (figura 1). Além dos índices dos critérios de qualidade de ajuste, a correlação entre os fatores não excedeu o valor de 0,80 e os indicadores de confiabilidade e validade se mantiveram dentro do esperado (tabela 2). $\mathrm{O}$ Alfa de Cronbach médio da escala foi de 0,83 .

Com relação à Escala de Intensidade de Busca de Emprego, foi considerada a estrutura bifatorial apontada por Wanberg et al. (2000), na qual a Escala de Intensidade de Contatos de Rede Social e a
Tabela 2

Confiabilidade e índices de validade da versão final das escalas

\begin{tabular}{lccc}
\hline \multicolumn{1}{c}{ Fatores } & $\alpha$ & CC & VME \\
\hline \multicolumn{4}{c}{ ESAT-BR } \\
$\begin{array}{l}\text { Dimensão da Realização } \\
\text { Pessoal do Trabalho }\end{array}$ & 0,86 & $0,970,90$ & $(0,94)$ \\
$\begin{array}{l}\text { Dimensão Positiva } \\
\text { do Trabalho }\end{array}$ & 0,84 & $0,930,80$ & $(0,89)$ \\
$\begin{array}{l}\text { Dimensão Negativa } \\
\text { do Trabalho }\end{array}$ & 0,78 & $0,800,64$ & $(0,80)$ \\
\hline
\end{tabular}

Escala de Intensidade de Busca de Emprego

$\begin{array}{llll}\begin{array}{l}\text { Intensidade de Contatos } \\ \text { de Rede Social }\end{array} & 0,88 & 0,970,86 & (0,92) \\ \begin{array}{l}\text { Intensidade Geral de } \\ \text { Busca de Emprego }\end{array} & 0,90 & 0,980,87 & (0,93)\end{array}$

Nota. $\alpha$ : Alfa de Cronbach; CC: Confiabilidade composta; vME: variância média extraída. Valores entre parênteses correspondem a raiz quadrada da VME.

Escala de Intensidade Geral de Busca de Emprego, são consideradas dimensões de um único modelo. Na primeira solução encontrada, apenas o $\chi^{2} / \mathrm{gl}=$ $3,564, p<0,001$, o SRMR $=0,053$, o CFI $=0,912 \mathrm{e}$ o GFI $=0,850$ se mantiveram dentro dos valores aceitáveis. O RMSEA $=0,107$ se manteve acima do valor esperado, enquanto que o TLI $=0,893 \mathrm{e}$ o AGFI $=0,787$, abaixo.

A partir desses resultados, com o intuito de aperfeiçoar o modelo, foram ligadas 5 duplas de erros, que também apresentaram alguma proximidade teórica e semântica. Após essas modificações, os índices passaram a apresentar os seguintes valores, sinalizando para a adequação do modelo: $\chi^{2} / \mathrm{gl}=$ $1,980, \mathrm{p}<0,001 ;$ RMSEA $=0,066$; SRMR $=0,043$; $\mathrm{TLI}=0,959 ; \mathrm{CFI}=0,969 ; \mathrm{GFI}=0,923 ;$ e $\mathrm{AGFI}=$ 0,882 (figura 2). Nenhum item foi excluído, pois todos apresentaram valores de carga padronizada superiores a 0,40. A correlação entre os fatores foi de 0,77 indicando para a capacidade discriminativa dos fatores. Quanto à confiabilidade e a validade, os valores estiveram dentro dos parâmetros estimados 


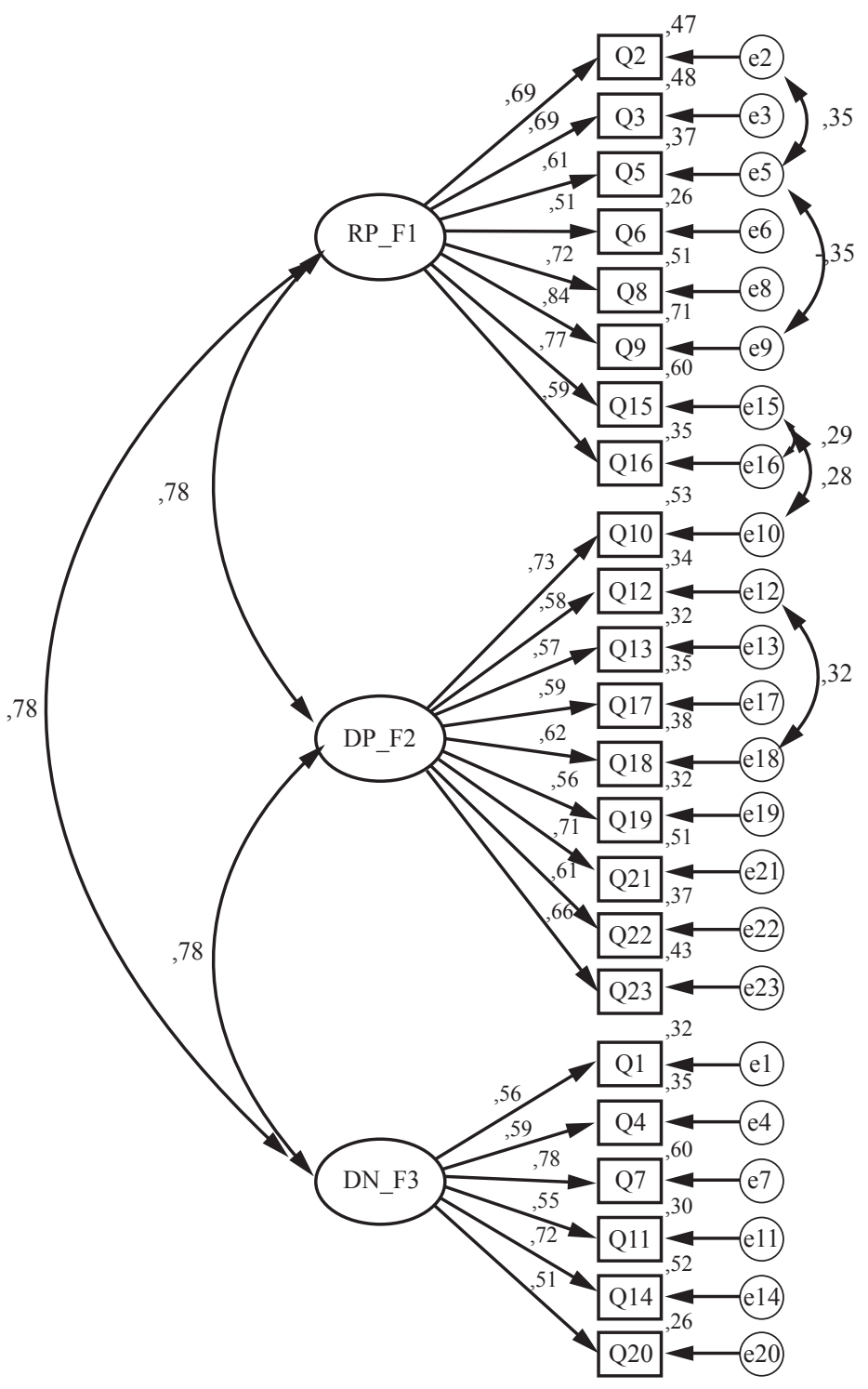

Figura 1. Modelo estrutural da ESAT-BR. RP_F1: Dimensão da Realização Pessoal do Trabalho; DP_F2: Dimensão Positiva do Trabalho; DN_F3: Dimensão Negativa do Trabalho

(tabela 2). O valor de Alfa de Cronbach médio da escala foi de 0,93.

Em seguida, foram realizadas análises descritivas com essas escalas. Os resultados evidenciaram que, quanto ao significado do trabalho, os escores tenderam a serem maiores na Dimensão da Realização Pessoal $(M=5,66, D P=0,63)$, e menores na Dimensão Negativa $(M=3,15, D P=$ 1,22). Quanto à centralidade relativa, o trabalho apresentou importância moderada, e figurou em segundo lugar $(M=19,97, D P=12,12)$, atrás da família $(M=39,80, D P=17,96)$, como segunda esfera de maior relevância. Já com relação à Escala de Intensidade de Busca de Emprego, os jovens relataram utilizar com maior frequência o uso de contatos de redes social em sua busca por uma ocupação $(M=2,30, D P=1,09)$.

No que diz respeito à associação entre os fatores das duas escalas, um dos principais objetivos deste estudo, constatamos que a Dimensão Negativa do 


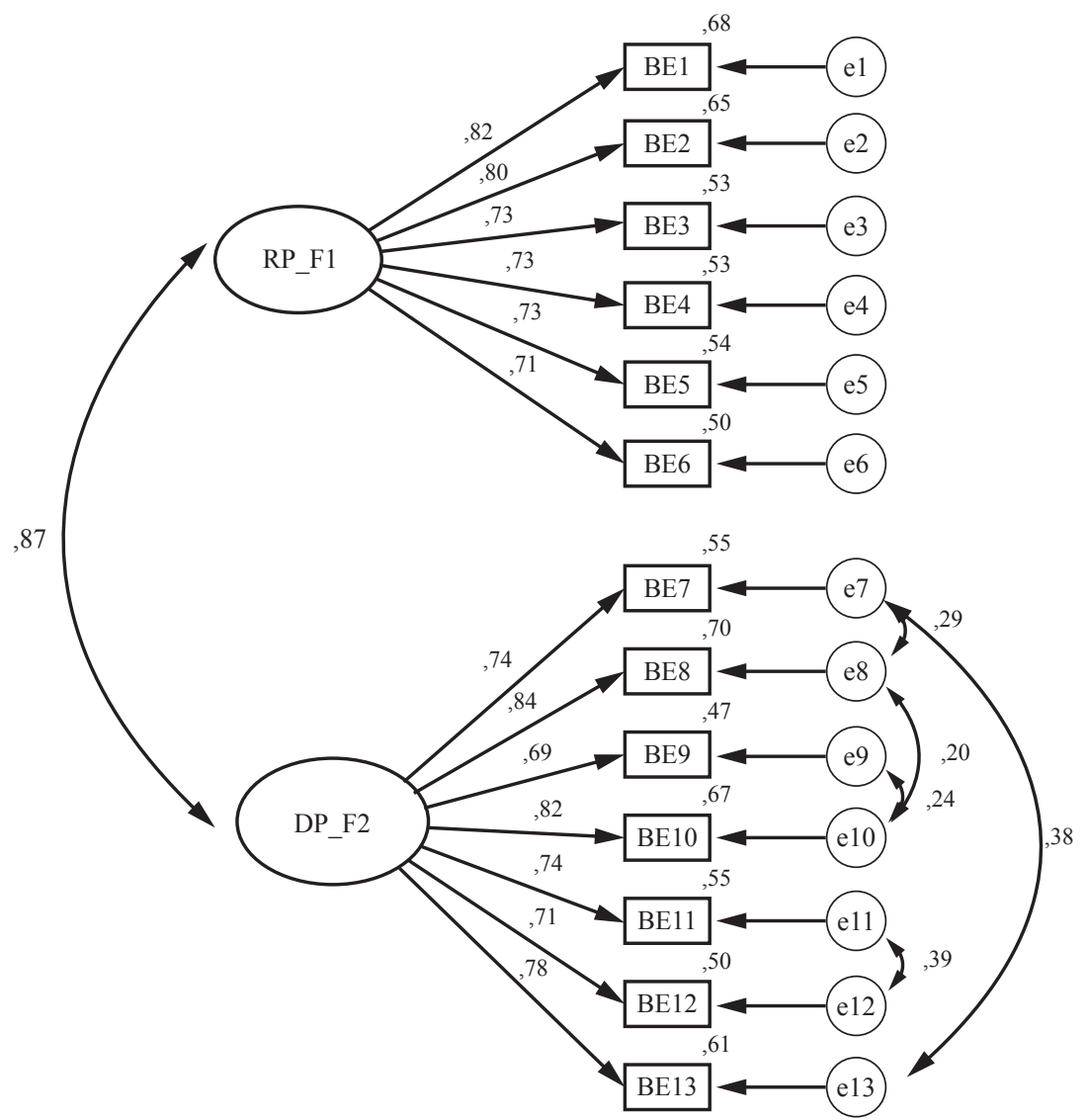

Figura 2. Análise fatorial confirmatória da Escala de Intensidade de Busca de Emprego. RS_F1: subescala de Intensidade de Contatos de Rede Social; SRS F2: subescala de Intensidade Geral de Busca Emprego

Trabalho esteve negativamente associada à busca de emprego com uso de redes sociais $(R S=-0,21$, $p<0,01)$, e sem o uso delas $(R S=-0,28, p<0,001)$. $\mathrm{O}$ relacionamento entre as escalas e as variáveis sociodemográficas, por sua vez, permitiu uma melhor compreensão sobre o fenômeno nem-nem, tal como estipulado nos objetivos do artigo. O tempo na condição, a renda e a escolaridade, estiveram relacionados com as dimensões do significado do trabalho e da busca de emprego (tabela 3).

Com relação ao significado do trabalho, jovens que estavam na condição entre 34 e 72 meses (Mdn $=3,50)$, atribuíram uma maior conotação negativa ao trabalho, comparativamente àqueles que estavam nela entre 15 dias e 9 meses $(\mathrm{Mdn}=2,83)$. O grupo de respondentes que estava na condição há mais tempo também fez menos referência à utilização de estratégias de busca de emprego. Jovens que estavam na condição entre 15 dias e 9 meses $(\mathrm{Mdn}=3,00)$, por exemplo, relataram utilizar com mais frequência a rede social como estratégia de busca, comparativamente aos respondentes que estavam nela entre 84 e 144 meses $(\mathrm{Mdn}=1,00)$. De forma semelhante, eles também se envolveram mais com outras estratégias de busca (15 a 9 meses, $\operatorname{Mdn}=2,28 ; 84$ a 144 meses, $\operatorname{Mdn}=1,00$ ).

Quanto à renda familiar, respondentes com rendimentos equivalentes a um salário mínimo e meio $(\mathrm{Mdn}=5,55)$ se diferenciaram dos que tinham renda de 20 a 30 salários $(\mathrm{Mdn}=4,55)$ na resposta à Dimensão Positiva do Trabalho. Similarmente, jovens com ensino fundamental incompleto (Mdn 
Tabela 3

Associação entre variáveis demográficas e dimensões das escalas

\begin{tabular}{|c|c|c|c|}
\hline \multirow{2}{*}{$\begin{array}{l}\text { Significado } \\
\text { do trabalho }\end{array}$} & $\begin{array}{l}\text { Tempo na condição } \\
\text { (34 a } 72 \text { meses) }\end{array}$ & Renda (1,5 salário) & \multirow[t]{2}{*}{$\begin{array}{l}\text { Escolaridade } \\
\text { (Fund. incompleto) }\end{array}$} \\
\hline & $\mathrm{U} \quad p \quad r$ & $\mathrm{U} \quad p \quad r$ & \\
\hline $\begin{array}{l}\text { Dimensão } \\
\text { Positiva }\end{array}$ & ---- & $\begin{array}{c}17,50 \quad 0,02 \quad-0,44 \\
r_{s}=-0,25, p<0,001 \\
\mathrm{H}(9)=20,35, p<0,05\end{array}$ & $\begin{array}{c}29,5 \quad 0,001 \quad-0,52 \\
r_{s}=-0,40, p<0,001 \\
\mathrm{H}(6)=37,13, p<0,001\end{array}$ \\
\hline $\begin{array}{c}\text { Dimensão } \\
\text { negativa }\end{array}$ & $\begin{array}{c}10310,02-0,21 \\
r_{s}=0,19, p<0,001 ; \mathrm{H}(3)=8,9320,35 \\
p<0,05\end{array}$ & & \\
\hline \multirow{2}{*}{$\begin{array}{l}\text { Busca de } \\
\text { emprego }\end{array}$} & $\begin{array}{l}\text { Tempo na condição } \\
\text { (15 dias a } 9 \text { meses) }\end{array}$ & $\begin{array}{c}\text { Renda } \\
\text { (8 a } 12 \text { salários) }\end{array}$ & $\begin{array}{l}\text { Escolaridade } \\
\text { (Fund. incompleto) }\end{array}$ \\
\hline & $\mathrm{U} \quad p \quad r$ & $\mathrm{U} \quad p \quad r$ & $\mathrm{U} p r$ \\
\hline $\begin{array}{l}\text { Com uso } \\
\text { de redes }\end{array}$ & $\begin{array}{c}750,001-0,46 \\
r_{s}=-0,30, p<0,001 ; \mathrm{H}(3)=20,52, p<0,001\end{array}$ & $\begin{array}{c}23,50 \quad 0,01 \quad-0,51 \\
r_{s}=0,32, p<0,001 \\
\mathrm{H}(9)=29,29, p=0,001\end{array}$ & $\begin{array}{c}246 \quad 0,001 \quad-0,62 \\
r_{s}=0,42, p<0,001 ; \mathrm{H}(6)=52,37, \\
p<0,001\end{array}$ \\
\hline $\begin{array}{c}\text { Busca } \\
\text { geral }\end{array}$ & $\begin{array}{c}67 \quad 0,000-0,49 \quad r_{s}=-0,35, p<0,001 \\
\mathrm{H}(3)=27,70, p<0,001\end{array}$ & $\begin{array}{c}17,50 \quad 0,00 \quad-0,63 \\
r_{s}=0,39, p<0,001 \\
\mathrm{H}(9)=43,57, p<0,001\end{array}$ & $\begin{array}{c}92,50 \quad 0,001 \quad-0,76 \\
r_{s}=0,60, p<0,001 ; \mathrm{H}(6)=91,42, \\
p<0,001\end{array}$ \\
\hline
\end{tabular}

Nota. rs: correlação de Spearman; H: teste de Kruskal-Wallis.

$=5,77)$ se diferenciaram daqueles com pós-graduação ( $M d n=5,00)$ na pontuação nesse fator.

Por fim, no que diz respeito às estratégias de busca de emprego, jovens cujas famílias concentravam os maiores rendimentos (8 e 12 salários, $\operatorname{Mdn}=4,00)$ se diferenciaram daqueles cuja renda era menos da metade de 1 salário $(\mathrm{Mdn}=1,58)$, quanto ao uso de redes sociais na busca de emprego, e sem o uso delas (Rendimentos de 8 a 12 salários, $\operatorname{Mdn}=3$; Menos da metade de um salário, Mdn =1,00). Já com relação ao nível de escolaridade, respondentes com ensino superior completo $(\mathrm{Mdn}=$ 3,33 ) relataram utilizar com mais frequência a rede social de contatos na procura por uma ocupação, do que jovens com ensino fundamental incompleto $(\mathrm{Mdn}=1,33)$. Da mesma forma, os respondentes com maior nível de escolaridade também se envolveram mais na busca de emprego por meio da utilização de outras estratégias (jovens com ensino superior, $\mathrm{Mdn}=3,28$; jovens com ensino fundamental incompleto, $\mathrm{Mdn}=1,00$ ).

\section{Discussão}

O primeiro objetivo desta pesquisa foi investigar a relação entre o significado do trabalho e os comportamentos de busca de emprego para jovens nem-nem. A partir das análises de correlação realizadas, foi possível identificar que jovens que atribuíram ao trabalho uma conotação negativa, se envolveram menos em comportamentos de busca de emprego. Especificamente, o tempo na condição auxilia a interpretar esse resultado, uma vez que longos períodos nela também estiveram associados a uma maior atribuição negativa ao trabalho e a um menor envolvimento nessa busca (Dorsett \& Lucchino, 2012).

No universo investigado, as experiências profissionais dos jovens podem influenciar a percepção negativa que eles têm sobre o trabalho. Em sua grande maioria, elas não foram registradas em carteira, tendo durado, em média, menos de um ano. Esses fatores, associados à exploração e 
pouco reconhecimento dos empregadores, poderiam explicar, ao menos em parte, tal percepção (Rocha-de-Oliveira et al., 2010).

O segundo objetivo da pesquisa foi o de identificar a centralidade do trabalho para o jovem na condição nem-nem. Sobre este objetivo, observou-se que o lugar atribuído ao trabalho possui um destaque moderado na vida dos jovens, corroborando o referencial teórico abordado, referente às mutações na importância atribuída ao trabalho na atualidade (Blanch, 2014; Harpaz et al., 2002). Na questão sobre a centralidade relativa, a família se destacou como a dimensão mais importante. No presente estudo, o fato de uma parcela considerável de jovens já possuírem filhos ou terem família própria, também explica essa valorização. Para eles, o trabalho é compreendido como meio de garantir os recursos financeiros que viabilizam, entre outras coisas, a vida familiar (Macêdo, Alberto \& Araujo, 2012).

A relação entre as características sociodemográficas com as dimensões do significado do trabalho e com os comportamentos de busca de emprego evidenciou que algumas variáveis pessoais e contextuais estão relacionadas a esses construtos. Em primeiro lugar, jovens de famílias que concentravam os menores rendimentos e aqueles com menor nível de escolaridade atribuíram uma conotação mais positiva ao trabalho. A interpretação para isto foi já antecipada na introdução: o trabalho se destaca como atividade importante para jovens de baixa condição socioeconômica pelo fato de proporcionar os meios de vida, de consumo e de esperança de transformação da sua realidade social e financeira (Dutra-Thomé \& Koller, 2014; Mota \& Tonelli, 2013). Muitos desses jovens abandonaram a escola precocemente para poder ajudar nas despesas domésticas, e tendem a se sentir mais produtivos no trabalho do que no contexto escolar. No entanto, questiona-se a qualidade das ocupações para as quais esses jovens são destinados - pois muitas delas são marcadas pela informalidade e precarização (Otero, 2011).
A renda e a escolaridade também estiveram associadas aos comportamentos de busca de emprego. Nesse caso, jovens que concentraram os melhores rendimentos e possuíam os maiores níveis de escolaridade relataram maior utilização de estratégias de busca. Esses resultados corroboram com os achados de Guimarães et al. (2012), de que a classe social possui relação com o tamanho da rede de contatos. Jovens de alto nível socioeconômico mantêm as maiores redes e têm acesso a informações sobre oportunidades de emprego de melhor qualidade. Por outro lado, assim como defendido por Kanfer et al. (2001) e Šverko et al. (2008), o menor nível de escolaridade está relacionado com o menor envolvimento com os comportamentos de busca, e uma das razões disso é a percepção da pessoa de que sua escolaridade não é suficiente para atender as exigências do mercado. No entanto, podemos destacar outros fatores de influência sobre esse pouco envolvimento com as estratégias de busca - entre eles, o acesso a informações e ao conhecimento sobre iniciativas governamentais de intermediação de mão-de-obra, que, em si mesmos, são uma das estratégias de busca de emprego possíveis.

Assim sendo, os resultados apresentados até aqui reforçam a importância de se compreender o fenômeno nem-nem a partir da junção de características individuais com o contexto de pertença desses jovens (Cardoso, 2013). Esta pesquisa possibilitou algumas aproximações nesse sentido. No entanto, seu caráter exploratório e transversal, baseado em entrevista estruturada (aplicação de questionário padronizado), não permitiu um aprofundamento nos aspectos psicossociais, e, sobretudo, biográfi$\cos$, associados à condição nem-nem. Tais elementos poderiam ser mais bem investigados por meio da análise das trajetórias de vida desses jovens, contemplando as duas esferas que compõem esse fenômeno: trabalho e educação.

Embora os resultados tenham explicitado que variáveis como tempo na condição, renda e escolaridade estão relacionadas com os motivos 
dos jovens estarem sem trabalhar, é preciso que outras investigações sejam conduzidas no intuito de compreender como se dá a relação entre essas variáveis e o fenômeno. Nesse sentido, as razões pelas quais os jovens de baixa condição socioeconômica se envolvem menos com comportamentos de busca de emprego, ou os motivos pelos quais jovens há mais tempo na condição atribuem uma maior conotação negativa ao trabalho, seriam mais bem explicitados. Com isso, haveria condições de se estabelecer vias científicas consistentes para compreender o fenômeno, que se apresentassem como alternativas ao discurso do senso comum, que recorre a uma pretensa falta de iniciativa dos jovens como motivo exclusivo deles estarem sem trabalhar e estudar.

Apesar dessas limitações, esta pesquisa permitiu uma aproximação teórica sobre um fenômeno investigado ainda de forma incipiente no contexto brasileiro (e mesmo internacional), sobretudo na psicologia do trabalho. Para essa área, que também tem se preocupado com as questões subjacentes à inserção dos jovens naquele mercado, a investigação do fenômeno pela via do significado e centralidade do trabalho, dos comportamentos de busca de emprego e das variáveis associadas a esses construtos, permitiu uma aproximação válida nesse sentido.

Espera-se que, a partir do presente estudo, outras questões sejam consideradas, influenciando a psicologia do trabalho e demais campos do conhecimento empenhados no estudo do tema. Neste sentido, sugere-se a importância de se analisar (ou de se priorizar como tema da pesquisa) a qualidade dos empregos assumidos, indo além da análise dos motivos dos jovens estarem sem trabalho. Dessa forma, a psicologia do trabalho poderá melhor se apropriar do seu objeto de estudo, estando sensível às questões laborais juvenis.

\section{Referências}

Balbinotti, M. A. A., Balbinotti, C. A. A., Marques, A. T. \& Gaya, A. C. A. (2009). Estudo fatorial confirmatório e da consistência interna do inventário do treino técnico-desportivo do tenista (ITTT-12). Revista Brasileira de Educação Física e Esporte, 23(4), 393-403.

Bendassolli, P. F., Alves, J. S. C. \& Torres, C. C. (2014). Inventário sobre significado do trabalho de profissionais de indústrias criativas. Avaliação Psicológica, 13(2), 177-186.

Benjet, C., Hernández-Montoya, D., Borges, G., Méndez, E., Medina-Mora, M. E. \& Aguilar-Gaxiola, S. (2012). Youth who neither study nor work: Mental health, education and employment. Salud Pública de México, 54(4), 410-417.

Blanch, J. M. (2014). La juventud NINI, un agujero negro psicosocial. Revista Psicologia: Organizações e Trabalho, 14(4), 355-366.

Brown T. A. (2014). Confirmatory factor analysis for applied research ( $2^{\mathrm{a}}$ ed.). New York: The Guilford Press.

Cardoso, A. (2013). Juventude, trabalho e desenvolvimento: Elementos para uma agenda de investigação. Caderno CRH, 26(68), 293-314.

Coelho, R. H., Estramiana, J. L. A. \& Luque, A. G. (2014). Juventud alargada y trabajo: Desafíos del mundo laboral en las experiencias de jóvenes brasileños y españoles. Revista Psicologia: Organizações e Trabalho, 14(4), 417-427.

Costa, J. S. M. \& Ulyssea, G. (2014). O fenômeno dos jovens nem-nem. Em C. H. Courseuil (org.), Desafios à trajetória profissional dos jovens brasileiros (pp. 114-136). Rio de Janeiro: IPEA.

Dorsett, R. \& Lucchino, P. (2012). Snakes and ladders in the youth labour market. Londres: National Institute of Economic and Social Research.

Dutra-Thomé, L. \& Koller, S. H. (2014). O significado do trabalho na visão de jovens brasileiros: Uma análise de palavras análogas e opostas ao termo 
"trabalho". Revista Psicologia: Organizações e Trabalho, 14(4), 367-380.

Escobedo, J. E. B. (2013). Juventud lapidada: el caso de los ninis. Nóesis. Revista de Ciencias Sociales y Humanidades, 22(44), 120-143.

Fernandes, F. S., Gonçalves, C. M. \& Oliveira, P. J. (2012). Adaptação e validação da Escala de Significados Atribuídos ao Trabalho (ESAT). Revista Brasileira de Orientação Profissional, 13(2), 183-195.

Field, A. (2009). Descobrindo a estatística usando $o$ SPSS ( $2^{\mathrm{a}}$ ed.). Porto Alegre: Artmed.

Gonçalves, C. M. (2006). A família e a construção de projetos vocacionais de adolescentes e jovens (Tese de doutorado não publicada). Universidade do Porto, Porto, Portugal.

Guimarães, N. A., Andrada, A. C. S., Picanço, M. F. \& Vieira, P. P. F. (2012). Trajetórias, atributos e relações. Representações sobre redes e obtenção de trabalho. REDES- Revista Hispana para el Análisis de Redes Sociales, 22(6), 106-146.

Hair, J. F., Anderson, R. E., Tatham, R. L. \& Black, W. C. (2009). Análise multivariada de dados ( $6^{\mathrm{a}}$ ed). Porto Alegre: Bookman.

Harpaz, I., Honig, B. \& Coetzier, C. (2002). A cross-cultural longitudinal analysis of the meaning of work and the socialization process of career starters. Journal of World Business, $37(1), 230-244$.

Harvey, D. (2009). Condição pós-moderna: Uma pesquisa sobre as origens da mudança cultural (18 ed.). São Paulo: Loyola.

Kanfer, R., Kantrowitz, T. M. \& Wanberg, C. R. (2001). Job search and employment: A personality-motivational analysis and meta-analytic review. Journal of Applied Psychology, 86(5), 837-855. DOI: 10.1037//0021-9010.86.5.837

Kanungo, R. N. (1982). Measurement of job and work involvement. Journal of Applied Psychology, 67(3), 341-349.

Kline, R. B. (2016). Principles and practice of structural equation modelling ( $4^{\mathrm{a}}$ ed.). New York: The Guildford Press.
Macêdo, O. J. V., Alberto, M. F. P. \& Araujo, A. J. S. (2012). Formação profissional e futuro: Expectativas dos adolescentes aprendizes. Estudos de Psicologia (Campinas), 29(Supl.), 779-787. DOI: http://dx.doi.org/10.1590/S0103166X2012000500014

Magalhães, M. O. \& Teixeira, M. A. P. (2013). Antecedentes de comportamentos de busca de emprego na transição da universidade para o mercado de trabalho. Psicologia: Teoria e Pesquisa, 29(4), 411-419.

Meaning of Work International Research Team (MOW) (1987). The meaning of working. Londres: Academic Press.

Mota, K. S. \& Tonelli, M. J. (2013). Percepções sobre trabalho entre jovens de diferentes estratos sociais. Anais do IV Encontro de Gestão de Pessoas e Relações de Trabalho. Brasília: Anpad.

Otero, A. E. (2011). Jóvenes estudiantes/jóvenes trabajadores: rutas desiguales, recorridos divergentes. Revista Electrónica de Investigación y Docencia (REID), 5, 175-195.

Rocha-de-Oliveira, S. R., Piccinini, V.C. \& Silveira, C. S. (2010). O trabalho como representação: A visão dos jovens universitários. Revista de Ciências da Administração, 12(28), 171-197. DOI: $10.5007 / 2175-8077.2010 \mathrm{v} 12 \mathrm{n} 28 \mathrm{p} 171$

Pais, J. M. (2016). Ganhos, tachos e biscates: Jovens, trabalho e futuro ( $4^{\mathrm{a}}$ ed.). Berlim: GD Publishing/Edições Machado.

Pochmann, M. (2007). A batalha pelo primeiro emprego: A situação atual e as perspectivas do jovem no mercado de trabalho brasileiro $\left(2^{\mathrm{a}}\right.$ ed). São Paulo: Publisher Brasil.

Schweitzer, L., Gonçalves, J., Tolfo, S. R. \& Silva, N. (2016). Bases epistemológicas sobre sentido(s) e significado(s) do trabalho em estudos nacionais. Revista Psicologia: Organizações e Trabalho, 16(1), 103-116. DOI: 10.17652/ rpot/2016.1.680

Seddon, F., Hazenberg, R. \& Simon, D. (2013). Effects of an employment enhancement programme on participant NEETs. Journal of Youth 
Studies, 16(4), 503-520. DOI: http://dx.doi.org/ 10.1080/13676261.2012.733808

Serracant, P. (2014). A brute indicator for a NEET case: Genesis and evolution of a problematic concept and results from an alternative indicator. Social Indicators Research, 117, 401-419. DOI: 10.1007/s11205-013-0352-5

Simmons, R. \& Thompson, R. (2014). Engaging young people not in education, employment or training: The case for a youth resolution. Londres: UCU.

Šverko, B., Galić, Z., Seršić, D. M. \& Galešić, M. (2008). Unemployed people in search of a job:
Reconsidering the role of search behavior. Journal of Vocational Behavior, 72, 415-428. DOI: 10.1016/j.jvb.2007.11.006

Vargas-Valle, E. D. \& Cruz-Piñeiro, R. (2012). Los jóvenes del norte y sur de México en inactividad laboral y educativa: niveles y factores asociados. Papeles de Población, 18(73), 1-43.

Wanberg, C. R., Kanfer, R. \& Banas, J. T. (2000). Predictors and outcomes of networking intensity among unemployed job seekers. Journal of Applied Psychology, 85(4), 491-503. DOI: 10.1037//0021-9010.85.4.491

\section{Recebido: agosto 29, 2016 Aprovado: novembro 18, 2017}

